DOCUMENTO

\title{
Trabajo Social InTERCULTURAL: Algunas Reflexiones a Propósito DE LA INTERVENCIÓN CON UNA COMUNIDAD INDÍGENA DEL TRAPECIO AMAZÓNICO COLOMBIANO
}

\author{
Por: Ruby León Díaz
}

\section{RESUMEN}

En este artículo se presenta un conjunto de reflexiones en relación a la manera cómo se realizó una intervención con la comunidad indígena de Mocaguab ${ }^{b}$ a propósito del impulso al Plan Integral de Vida (PIV). Se trata de analizar tres dimensiones que encuadran dichas reflexiones en el antes, el durante y el después de la intervención: en primer lugar se abordará la dimensión conceptual de la intervención intercultural que contiene a su vez categorías discursivas y políticas que justifican la acción profesional. En segundo lugar se examinará la dimensión metodológica que da cuenta de la práctica profesional en sí misma, la cual trasciende la mera técnica pues desde allí se involucra no solamente a los sujetos con los cuales se construye el entramado interventivo sino también el sujeto profesional. Por último se planteará una mirada a la dimensión ética del impulso de un Plan Integral de Vida en una comunidad indígena, pues desde allí es posible cuestionar propuestas conceptuales y metodológicas homogenizantes sobre la manera de ver lo indígena. Los Plan Integral de Vida son un excelente escenario para estudiar la forma cómo se concibe la inclusión de la diversidad étnica a la Nación. Sin demeritar la importancia de la elaboración de los Plan Integral de Vida por parte de las comunidades, es posible cuestionar la idea de respeto a la diferencia cultural que en ellos prevalece, máxime cuando dichos Plan Integral de Vida no problematizan de manera frontal las desigualdades y la exclusión social histórica de las comunidades indígenas.

Palabras Claves: intervención, Trabajo Social Intercultural, Planes de Vida, comunidades indígenas, metodología, ética.

\section{SUMARY}

This article presents a set of thoughts about the way was made an intervention with indigenous community-Mocagua, referit to impulse the Plan Integral de Vida. 
The principal objective is to analyze three dimensions before, during, an after the intervention. In first moment, I present the conceptual dimension of the intercultural intervention that contains polities and discursive categories as a justification of the professional action. In the second part, I will examine the methodological dimension that tackle the professional practice in itself, its go beyond the simple technical aspect, and refers to subjects involves on the interventive network and to the professional subject. Finally I present to the ethical dimension about the impulse of a Plan Integral de Vida with an indigenous community, because with this is possible to debate conceptual and methodological hegemonic proposals about the way it is understood the indigenous identity. The Plan Integral de Vida is an excellent setting to study the mode it is conceived the inclusion of the ethnic diversity in our Nation. Without reducing the importance of the building PIV, it is possible to debate the idea of respect to the cultural difference of this special communities, especially when the Plan Integral de Vida do not attack frontally the inequalities and the historical social exclusions of the indigenous communities in Colombia

\section{Key Words: Intervention, Intercultural Social Work, Planes Integrales de Vida, Indigenous Communities, methodology, ethic.}

\section{Más Allá de la Contemplación de la Diferencia Étnica: Apuntes Desde el Trabajo Social Intercultural.}

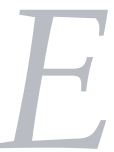

Trabajo Social como profesión que procura la justicia social para garantizar la dignidad humana, ha concentrado su quehacer en relación con los problemas sociales, frente a los que el Trabajo Social se ve abocado a intervenir guiado por un sentido ético que aboga por la construcción de la condición humana digna ${ }^{c}$. Sin embargo es de admitir que esta intervención sobre la realidad social ha estado permeada por proyectos ideológicos y de sociedad, que de manera conciente o no han promovido modelos ideales sobre cómo ser individuo, cómo debe ser la familia y qué debe ser una comunidad. Dichos modelos se relacionan con arquetipos europeos o andinocéntricos ${ }^{\mathrm{d}}$, algunos de los cuales buscan normalizar conductas y formas de vida consideradas atípicas comparadas con la imagen de la nación mestiza, de familia nuclear, religión católica, lengua castellana, entre otros modelos ideales hegemónicos. Desde una mirada crítica al Trabajo Social hegemónico, se puede afirmar que éste ha hecho eco a los modelos ya enunciados. Sin embargo un Trabajo Social que se ubique al lado de los grupos y comunidades que se alejan de estos ideales hace que emerjan una serie de cuestionamientos a las prácticas y discursos profesionales desde intervenciones que buscan comprender la diversidad étnica. Para emprender este camino se necesita adentrarse en el Trabajo Social intercultural.

Palubrano. 8 Agosto/2007 
La reflexión intercultural llega a nuestra disciplina para entender aquellos procesos que al no encajar en la tipificación de los problemas sociales "clásicos", deben atenderse como variaciones del proceso de intervención profesional. Mary Richmond, aunque desde la corriente positivista, es quien inicialmente considera la importancia de la variable cultural en la atención a familias de inmigrantes; sugiriendo además que al enfrentar este tipo de realidad el/la profesional debe "aprender que no puede ignorar las características nacionales de los inmigrantes por completo; esto para no caer en el error de considerarlos como miembros de una colonia o de una nacionalidad, con unas características fijas; y tampoco en el error de ignorar sus peculiaridades nacionales raciales y tratar de aplicarles los mismos criterios que aplicaría a sus compatriotas"e.

En análisis más recientes sobre la intervención social que resalta la diferencia cultural algunos autores como Octavio Vázquez consideran que la interculturalidad "propone un marco de actuación coherente con la filosofía y los principios tradicionales de la disciplina de Trabajo Social, ya que fomenta el desarrollo de la comunicación, el conocimiento del Otro, la valoración de sus aportaciones, la negociación y el establecimiento de objetivos en común”․ Este autor también sugiere que en el encuentro profesional con Otros diversos, además de las diferencias culturales evidentes (el idioma y la religión por ejemplo), también se presentan una serie de distancias estructurales que dan cuenta de la diferencia como sinónimo de desigualdad social, cultural y económica. Las desigualdades estructurales se vuelven visibles en las condiciones básicas de sobrevivencia o en la insatisfacción de necesidades vitales ${ }^{\mathrm{g}}$. El Trabajo Social intercultural se ocupará entonces de crear

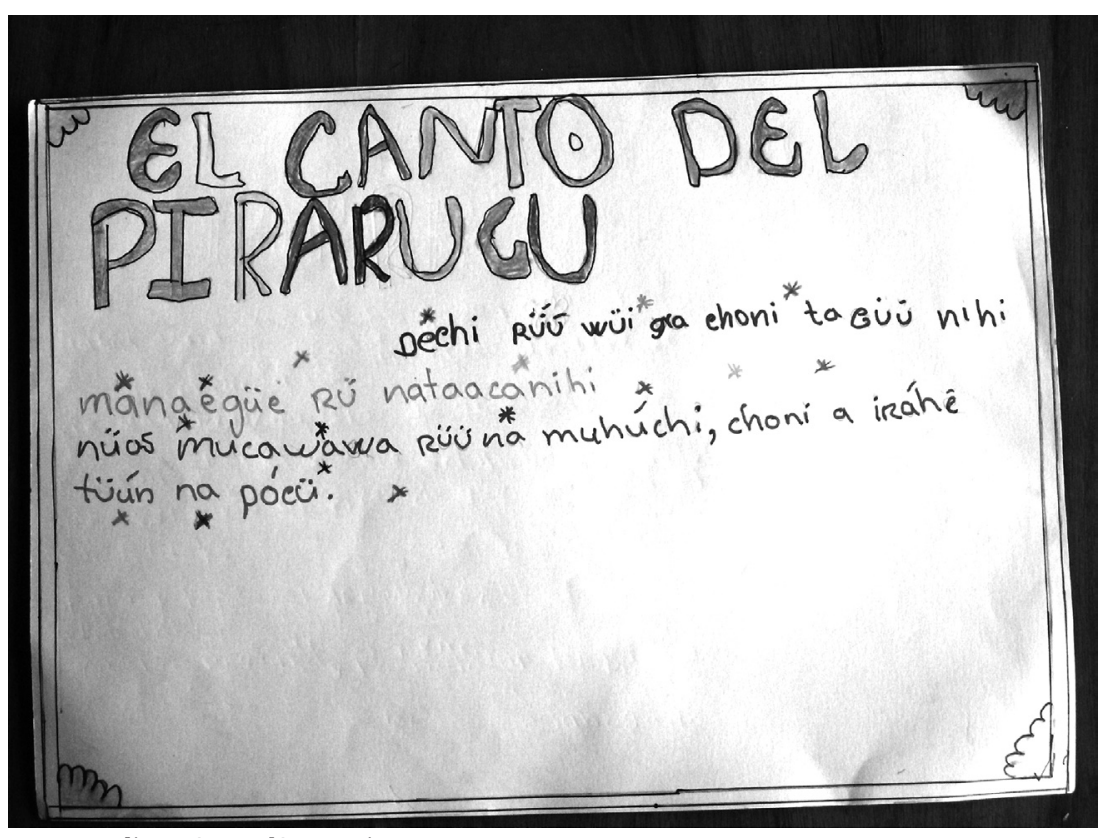
condiciones para que una sociedad que se considera éticamente correcta y adscrita a valores democráticos como la libertad, la igualdad, la fraternidad, la justicia, los derechos humanos, la multiculturalidad y la plurietnicidad actúe en consonancia. Por ello cuando el Trabajo Social se preocupa por la diversidad étnica lo hace desde una apuesta ética para la transformación de la perversa ecuación entre diferencia cultural y desigualdad social. Es la actuación sobre esta ecuación lo que justifica la intervención de Trabajo Social en Foto: Julia León, Indígena Ticuna. 
una realidad étnica y culturalmente diversa. No corresponde a esta disciplina la contemplación de la diferencia por la diferencia.

Al reconocer la particularidad de la realidad sociocultural de los Otros en cuanto a diversidad étnica y a desigualdad estructural, a nivel profesional surge la reflexión sobre la competencia intercultural, que como estrategia para la acción profesional es fundamental en la comprensión de la intervención desde la acción y también desde la reflexión en contextos étnicamente diferenciados. Más allá de ser una habilidad, un instrumento o una técnica, lo que aquí entendemos por competencia intercultural es "una relación que se establece entre sujetos diferentes (...) que permite a los profesionales ser capaces de comunicarse con el Otro diferente, para lograr captar los significados que se otorgan a los elementos que se comparten (...), teniendo en cuenta que comprender, desde la óptica intercultural, equivale a identificar las razones que explican el comportamiento del Otro, sin que esto implique aceptar, justificar y/o descalificar automáticamente sus razones" h

Para resaltar la sensibilidad intercultural en el proceso de intervención es necesario desarrollar tres competencias básicas, como las llama Octavio Vázquez. La primera, es la competencia cultural relacionada con la capacidad de negociación con los significados culturales de los Otros, que sirve para motivar la interacción. La segunda es la competencia cognitiva entendida como la capacidad de conocimiento cultural que se tiene sobre el Otro y sobre sí mismo que permite definir los sentidos a intercambiar, la cual se traduce por ejemplo en "reformular los problemas desde la óptica del sujeto con su cultura aunque las explicaciones sean inadecuadas desde la perspectiva occidental" ${ }^{\prime}$. Por último está la competencia emotiva, como aquella capacidad de emocionarse antes, durante y después de la relación intercultural con el Otro, lo que se traduce por ejemplo en el logro de la empatía.

Adicionalmente, citando a Esteban Ruiz, se podría considerar que para que estas comprensiones o competencias básicas de la intervención intercultural emerjan debe existir una propuesta no hegemónica del sujeto profesional respecto al sistema de poder en el que se inscribe. En una propuesta no hegemónica de intervención surgen representaciones plurales del Otro y su situación, como imágenes que buscan empoderar y reconocer a los Otros como sujetos y no sólo como entes receptores de la acción. Lo que implica, según Esteban Ruiz "la construcción colectiva de una representación propia que se entiende como motor de una transformación de las relaciones de poder dentro de la intervención social, habilitando a los usuarios como sujetos y no ya como meros objetos del proceso" ${ }^{\prime \prime}$. Esta imagen plural abre el camino a una intervención participativa, que gestiona el empoderamiento, al estar basada en la 
horizontalidad de las relaciones entre agentes e intervenidos, y se vuelve auténtica cuando "implica a la gente tanto en fijar los asuntos y temas sobre los que intervenir, en el uso de los recursos, así como en el control sobre el conjunto del proceso de intervención (...). La intervención así concebida precisa una predisposición en profesionales y políticos, así como un conocimiento, sensibilidad y compromiso de todos ellos con la estrategia”k

Además de la intervención participativa y las representaciones plurales del Otro y su situación la competencia intercultural también contribuye a reconocer a los sujetos involucrados en la acción profesional, como sujetos inscritos en una cultura, y a establecer puntos de contacto o espacios para negociar, tanto desde la razón como desde la emocionalidad de cada una/o de las/os involucradas/os en la relación, para desde allí fijar los objetivos de la intervención intercultural.

Situar la competencia intercultural en la intervención permite trascender la idea de la acción profesional como conjunto de acciones dispersas sobre un(os) sujeto(s) que se considera(n) culturalmente diferente(s) y en términos sociales desigual(es) hacia la idea de un accionar reflexivo sobre la necesidad de comprensión cultural de la diferencia como diversidad y no como desventaja. Es reconocer que en la intervención no se produce simplemente conocimiento cultural unidireccional que parte del profesional, sino que es una relación en la que se presenta un intercambio cultural dinámico e interactivo. Por lo tanto la competencia de los y las profesionales en Trabajo Social con respecto a la intervención intercultural parte de tomar conciencia de la diversidad cultural y de la dinámica de cambio que genera cualquier comunicación, sin olvidarse que el simple hecho de poner en contacto a personas diferentes en un mismo espacio no presupone garantía de éxito en el intercambio"l ${ }^{1}$, así como tampoco supone que el Otro es el único portador de la cultura y de la diferencia.

Reconocer la "imperfección" del intercambio cultural en la intervención permite plantear reflexiones-acciones más justas respecto al sujeto (en este caso la comunidad indígena) a quien va dirigida nuestra acción, al tiempo que sobre el sí mismo como sujeto interviniente, también diverso para el Otro. Esto nos permite evitar reflexiones y actuaciones en las que se pierde de vista a los sujetos que "deciden aceptar" la intervención, esgrimiendo el argumento de la asimilación en valores de la Democracia (por ejemplo, el Progreso, el Bienestar, el Desarrollo, la Integración, la Participación, la Planeación Participativa, etc.). Otro riesgo que se puede encarar con la reflexión sobre la intervención es que en la acción se pierdan los sujetos profesionales, que al identificarse de manera absoluta 
al Otro étnicamente diferente, olvidan que a pesar de la intensidad de los acercamientos culturales difícilmente podrán fundirse o pretender representar y comprender por completo la cosmovisión del Otro, puesto que étnica y culturalmente también vehiculan una cultura generalmente más cercana a la hegemónica.

A diferencia del caso norteamericano y europeo $^{\mathrm{m}}$ en el cual el componte étnico-intercultural se instaura desde una alteridad radical $^{\mathrm{n}}$ entre profesional (nacional) y sujeto (inmigrante), en el contexto de América Latina y el Caribe la cuestión de la diferencia desde lo étnico cultural difícilmente se resuelve al trazar una línea divisoria entre "ellos" (extranjeros) y "nosotros" (nacionales). Pues esta diferencia cultural hace parte constitutiva, aunque no siempre de manera explicitada, de las identidades nacionales

\section{Trabajo Social y la Cuestión Cultural Étnica en la Intervención Profesional}

La reflexión académica sobre la intervención profesional desde Trabajo Social sensible a la diferencia étnico-racial ${ }^{\circ}$ empieza a desarrollarse de manera reciente en nuestro país. A continuación se referencian tres textos que dan cuenta de la reflexión en torno al reconocimiento sobre la alteridad étnico-racialmente diferenciada en el proceso de intervención.

Una reflexión inicial es desarrollada, a propósito de la experiencia de intervención con comunidades indígenas, por Rocío Ángel, Gilma Pérez, Martha Rico, Rosa Sandoval y Cecilia Vargas en 1974: "Acción de Trabajo Social en el campo indígena". Este trabajo fue adelantado desde la Universidad Social Católica de La Salle, con el objetivo de comprobar, desde una experiencia práctica de trabajo con indígenas Embera del Cauca, "la factibilidad de la acción de Trabajo Social con comunidades indígenas"p. A partir de la descripción del contexto cultural y geográfico de la intervención, la elaboración de algunas referencias teóricas sobre el trabajo comunitario, los métodos de caso, grupo y comunidad, y el planteamiento de la metodología de intervención (observación, visita domiciliaria, test, encuestas, entre otras), las autoras describen cómo por medio de instrumentos propios del Trabajo Social es posible intervenir con comunidades indígenas. Con este trabajo se evidencia el interés por la intervención de la profesión con comunidades indígenas en Colombia que se funda en el análisis antropológico, al ser el único insumo teórico considerado por las autoras para la compresión de lo que ellas denominan el "campo indígena". 
Existen otros dos artículos recientes que dan cuenta de reflexiones sobre la relación intercultural. El primero se intitula "La intervención institucional en contextos culturalmente distintos: lógicas en tensión y contradicción" escrito por Martha Bello y Constanza Millán ${ }^{q}$, en el que referencian procesos de intervención institucional en contextos culturalmente distintos y se traza una panorámica general acerca de las lógicas de acción y tensiones presentes en la práctica profesional institucionalizada.

El segundo texto es "Pluralismos epistemológicos: hacia la valorización teórica de los saberes de acción" de Claudia Mosquerar ${ }^{\mathrm{r}}$ quien, entre otros temas, (saberes de acción, intervención, profesiones relacionales) se propone una reflexión sobre el concepto de interculturalidad como "el complejo histórico de relaciones asimétricas entre actores culturales diferentes(...) que surgen en la relación intersubjetiva que se da en el encuentro mediante el diálogo, la confrontación o la imposición entre dos personas que representan algún tipo de cultura"s. Esta concepción de lo intercultural aporta dos aspectos importantes: uno es el carácter de la asimetría en el encuentro intercultural; y el otro aspecto se refiere al reconocimiento de que la intervención como relación intersubjetiva no sólo se puede dar de manera concertada o dialógica sino también desde la confrontación y/o la imposición.

Se resaltaron en particular estos dos textos debido a que ilustran el interés por reflexionar sobre la intervención en contextos que convocan atención especial a sujetos étnico-racialmente diversos. En seguida presentaré una propuesta de comprensión respecto del Trabajo Social Intercultural, a propósito de la experiencia interventiva con una comunidad indígena del Trapecio Amazónico Colombianot.

\section{Propuesta de Compresión Sobre el Trabajo Social con Comunidades Indígenas}

Con el objetivo de reflexionar respecto de la intervención de Trabajo Social en contextos étnicamente diversos en el marco de valores democráticos que se cristalizan en Derechos Humanos étnicos que para el caso de los Planes Integrales de Vida se relacionan con el reconocimiento de "las aspiraciones de los pueblos indígenas, de sus propias instituciones y formas de vida y de su desarrollo económico" dentro del Estado colombiano ${ }^{\mathrm{u}}$, adelantamos una intervención en la Comunidad indígena de Mocagua con el objetivo de dar impulso y colaborar en la construcción del Plan Integral de Vida comunitario. Después de reflexionar 
sobre las particularidades de noción de intervención intercultural queremos proponer una manera de compresión sobre la acción profesional en una realidad étnica distinta. A continuación se presenta una reflexión colectiva ${ }^{v}$ sobre el encuentro intercultural en la intervención desde Trabajo Social.

- Dimensión Conceptual de la Intervención Intercultural: Comprensión del Contexto Cultural, Discursivo y Político para la Intervención Profesional

Algunos autores proponen que al iniciar un proceso de intervención intercultural ${ }^{\mathrm{w}}$ es importante lograr cierta sensibilidad que permita reconocer encuentros y desencuentros culturales entre los sujetos involucrados en esta relación. Siguiendo la propuesta de Esteban Ruiz ${ }^{x}$ respecto de la intervención social, comprendemos que la importancia de la construcción de la acción profesional en ámbitos culturalmente diversos se da a partir de tres elementos: el cultural, el discursivo y el de las relaciones de poder.

El reconocimiento del elemento cultural en primer lugar implica visibilizar rasgos de las identidades indígenas en comunidades concretas, la presencia de la diversidad en la diversidad, así como también encontrar espacios que permitan comunicarse con el Otro, para desde allí proponer acciones que involucren a los sujetos adscritos a la intervención.

El segundo elemento conceptual que es necesario comprender es el discursivo, que permite el extrañamiento respecto

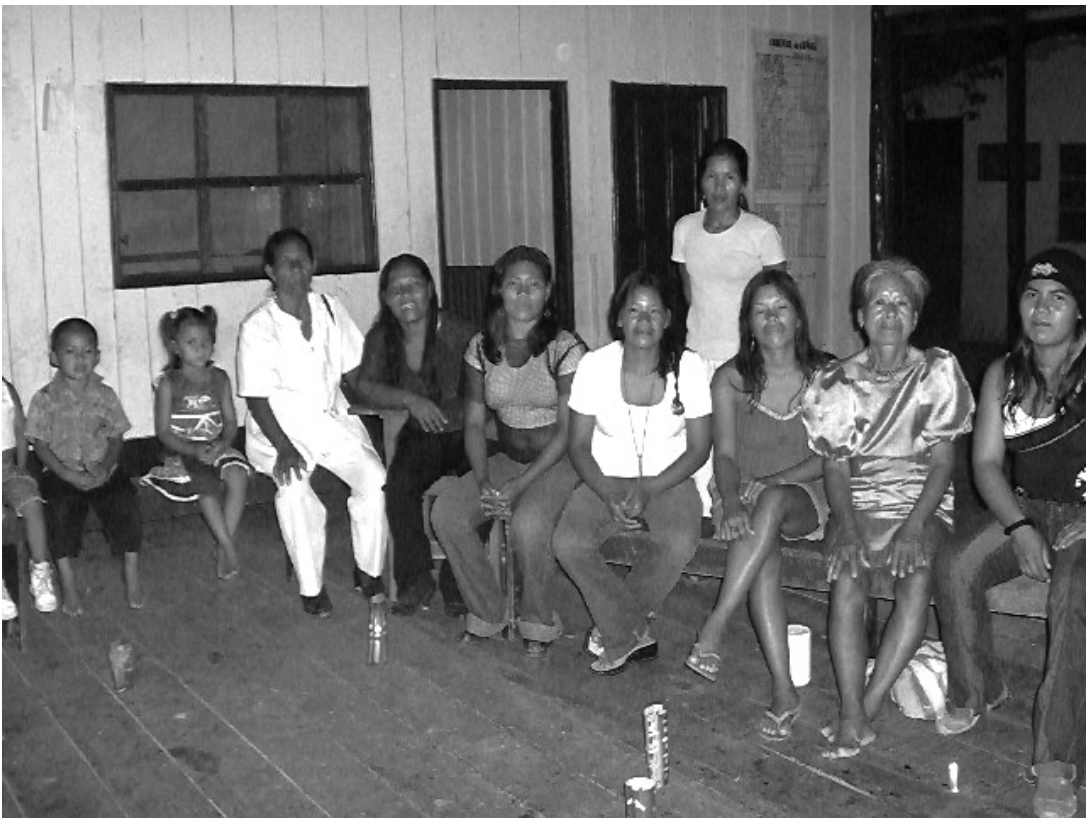

Foto: Julia León, Indígena Ticuna. de una serie de ideales hegemónicos, desde nuestra experiencia particular, conceptos como democracia participativa, multiculturalidad, plurietnicidad y desarrollo, justifican la ingerencia en espacios particulares. Quien interviene debe decidir si toma tales ideales para justificar de manera dogmática la pertinencia de su acción profesional o, en cambio, relativiza la materialización de dichos imperativos democráticos en la realidad de comunidades indígenas, al encontrar, como nos ocurrió, que desde los ideales de nación mestiza siguen siendo imaginadas por la Nación colombiana, como "lejanos habitantes de territorios que históricamente escapan al proyecto de integración nacional”y. 
El tercer elemento que permite la comprensión de la intervención intercultural se refiere a lo político, elemento que conjuga lo cultural, lo discursivo y las relaciones de poder en el nivel micro-relacional; esto es, de sujeto (profesional de la intervención) a sujeto (indígena), uno y otro inmersos en un contexto mediatizado por relaciones de poder a un nivel más amplio.

Lo importante del reconocimiento de este conjunto de elementos conceptuales que hemos agrupado en la comprensión de la dimensión conceptual para la acción por parte de quien interviene sobre y desde una realidad cultural particular, es aspirar formalmente a la coherencia entre lo que discursivamente se promueve (por ejemplo la valoración y respeto por el Otro en su diferencia) y el ejercicio profesional en concreto. Esto en la práctica permite no caer en juicios que descalifican, y que se evidencian en interpretaciones y juicios relacionados con la idea de aculturación y pérdida identitaria para referirse a los cambios culturales que han vivido, y que continúan viviendo, algunas comunidades indígenas. Se trata entonces de que quienes aspiran a intervenir sobre una realidad étnica diferente construyan una representación que "sea motor de transformación de las relaciones de poder dentro de la intervención social, habilitando a las personas como sujetos y no como meros objetos del proceso" ${ }^{\prime \prime}$.

Nuestra propuesta interventiva fue el impulso al Plan Integral de Vida (PIV) de la comunidad indígena de Mocagua, considerada a pesar de las múltiples críticas a los PIV, especialmente referidas por un lado a la reducción que sufre la cosmovisión indígena al plantear en su escritura solamente proyectos técnicos, y por otro lado la crítica a la autonomía ligera ${ }^{\text {aa }}$ que se concede a las comunidades en relación con la toma de decisiones y manejo de sus recursos. Desde nuestra práctica comprendimos que los PLAN INTEGRAL DE VIDA pueden llegar a ser un aporte para la reivindicación étnica y el reconocimiento histórico de luchas políticas; para lo cual deben estar vincularlos a las asociaciones, organizaciones y comunidades indígenas para la discusión política por el reconocimiento en niveles nacionales y supranacionales.

Con el objetivo de "impulsar a la formulación del Plan Integral de Vida...", propusimos en terreno una serie de actividades orientadas a consolidar cierta información sobre problemáticas comunitarias desde distintos grupos de población, y a la vez propiciar una reflexión sobre la importancia de trasmitir la historia reciente de la comunidad. Otra actividad estuvo relacionada con la consolidación de un censo comunitario, que fue construido a partir de una serie de formatos que la Corporación para el Desarrollo de la Biodiversidad Amazónica (CODEBA) nos facilitó. 
Por último, también desarrollamos un conjunto de actividades con niños y niñas desde la escuela con dos fines: el primero fue la devolución de la información que habíamos logrado en las otras actividades, la cual se relacionaba en particular con la historia comunitaria; el segundo reconstruir parte de la historia reciente de la comunidad teniendo como base los relatos y vivencias personales y familiares de mujeres y hombres adultos de la comunidad. También hubo una serie de actividades que algunos grupos comunitarios nos propusieron. La labor en estos espacios fue de acompañamiento a la dinámica grupal-sobre todo con las mujeres del restaurante- y en la trascripción de ideas sobre papel, como por ejemplo la redacción de proyectos para solicitud de materiales o propuestas eco-turísticas.

En resumen, a partir de la intervención situada fue posible reconocer que parte de nuestra opción discursiva, política y cultural estuvo antecedida por una noción idealizada de los Planes Integrales de Vida que partía del hecho de considerar que: en primer lugar que dichos Planes eran como la única estrategia para lograr el reconocimiento del pensamiento y la cosmovisión indígena por parte del Estado; en segundo lugar que debía construirse con "todos", sin tener en cuenta sus dinámicas internas y las relaciones de poder y jerarquía que se tejen en su interior y que fundamenta la noción de futuro de la comunidad. Por fortuna en el ejercicio práctico logramos dilucidar matices de esas ideas y construir otra forma de intervención de acuerdo con las demandas y realidades organizativas de la comunidad indígena de Mocagua.

- La Dimensión Metodológica de la Intervención Intercultural: un Asunto que Trasciende la Pragmática de la Intervención

La metodología en Trabajo Social remite al conjunto procesos que nos permiten alcanzar determinados objetivos de la intervención ${ }^{\mathrm{ab}}$. En el periodo de la Reconceptualización sobre el método de acción (individual y de grupo) sus técnicas derivadas se consideraban puntuales, desarticuladas y pro-hegemónicas. Existieron múltiples críticas al considerar que, amparándose en unas fases o momentos del trabajo profesional, lo que se buscaba con la intervención era contener, regularizar o normalizar a individuos que tenían una serie de problemáticas desestabilizadoras del sistemac.

En cambio hoy encontramos un resurgir de la metodología para la intervención, no sólo desde Trabajo Social o Psicología, sino desde otras disciplinas de las Ciencias Sociales, como un conjunto de herramientas importantes que permiten fijar los postulados teóricos de la intervención en la realidad. Por metodología para la 
intervención social entendemos el conjunto de acciones que buscan "marcar el contenido pragmático de la intervención para producir cambios que afecten a las personas"ad.

Como el concepto mismo de metodología remite a un conjunto de instrumentos y técnicas, es importante sujetar esas acciones puntuales a reflexiones epistemológicas que las orienten, así como evidenciar la dimensión ética que dirige su finalidad. Sin estos elementos el quehacer práctico queda reducido a lo que Alipio Sánchez denomina tecnocracia, en la cual lo importante son los resultados y no el proceso en sí mismo ${ }^{\text {ae }}$.

El ser concientes de que la metodología por sí sola no hace a la intervención social y mucho menos construye Trabajo Social, nos obliga como profesionales a proponer la sincronía entre la reflexión ética, la reflexión conceptual y la reflexión sobre el contexto histórico, político, económico y cultural en el cual se desarrolla la acción profesional. A su vez implica tomar en serio lo metodológico para no caer en prácticas, ya antes criticadas, que enfatizan en los medios y no en las finalidades de la intervención, y también con el fin de tomar opción por una coherencia interventiva (conceptual, ética y metodológica) que garantice y obligue a la responsabilidad política y social por las acciones adelantados por las/os sujetos profesionales.

Retomando la dimensión intercultural de la intervención social en relación con la metodología, Belén Lorente propone una serie de consideraciones especiales en torno a la actuación de Trabajo Social, que a nuestro parecer facilitan la interconexión de la dimensión metodológica con los niveles conceptual y ético de la reflexión, sin perder de vista la diferencia como diversidad cultural del sujeto a quien se dirige la intervención. Citando a Cohen, Belén Lorente propone un esquema de la acción que incluye tres tareas, que creemos pueden ser leídas como principios metodológicos. La primera es la descentración del sí mismo cultural, en el cual "apropiarse del paradigma de la relatividad cultural -todas las culturas tiene igual valor- no significa perder la propia identidad o pensar que todo vale" af. La segunda es la penetración en el sistema del Otro, que implica informarse acerca de la realidad cultural del Otro. En tercer lugar, está la negociación/mediación que impele al profesional a "reconocer que al haber un conflicto de valores en la consideración del Otro étnicamente diverso como interlocutor válido, los cambios y aproximación deben darse desde ambos sentidos y no esperar que el Otro sea el que cambie"ag. 
La propuesta de Belén Lorente es importante para la reflexión sobre la dimensión metodológica ya que plantea la necesidad de dar participación sustantiva al sujeto (diverso) en la intervención social, lo que implica restituirlo como coproductor y diríamos como alter -cognoscente y emocional- del profesional en el nivel metodológico de la intervención. Esas tareas o principios nos indican que los componentes de la dimensión metodológica tienen una estrecha relación con la ética.

Además de estos principios para la acción, consideramos que otro elemento metodológico importante se encuentra en el nivel de la acción propiamente dicha. Existen unas formas de actuar sobre la realidad (con individuos, grupos y comunidades) que se presentan como herramientas metodológicas, en las que además de lo específico -la técnica misma, por ejemplo observación participante, taller, visita domiciliaria-, se involucra lo discursivo, las relaciones de poder y lo cultural.

En resumen, propusimos en campo una serie de actividades puntuales, relacionadas con una metodología para acción, que hacían parte de un proceso orientado a dar cumplimiento al proyecto de impulso al Plan Integral de Vida y a acompañar iniciativas de grupos comunitarios que solicitaban una labor de traducción -por así decirlo- del lenguaje comercial del ecoturismo (costeo de productos, por ejemplo). Otro conjunto de herramientas fueron los medios para la autorreflexividad, para conocernos y acercarnos a nuestra propia cultura, los cuales permitieron también reencontrarnos con el Otro, indígena contemporáneo en concreto, y valorarlo en su diversidad de pensamiento, manejo de su entorno y su visión de mundo.

- La Dimensión Ética de la Intervención Intercultural

Las valoraciones y postulados éticos anteceden, moldean y direccionan la intervención profesional. Por eso es importante que quienes deciden actuar sobre realidades diversas, además de la conciencia sobre la cultura propia y la del Otro -como sugeríamos atrás-, también tengan en cuenta que la intervención es un acto ético en la medida en que implica un ejercicio profesional y personal en el que existe una conciencia moral con cierto grado de libertad, que a su vez le permite asumir al sujeto profesional una responsabilidad, no sólo respecto de sus opciones y acciones, sino sobre todo respecto a cómo utiliza el poder que la sociedad y sus instituciones ponen en sus manos, ya que la intervención siempre tiene una intencionalidad hacia los Otros "heterogéneos, diferenciados y que están socialmente interrelacionados"ah.

Palobrano. 8 Agosto/2007 
Alipio Sánchez entonces propone que, además de darse una reflexividad ética orientada a "examinar la bondad o maldad de las distintas opciones y responsabilidades profesionales derivadas de ellas, en la intervención también debe existir una reflexión y valoración de los intereses políticos que implican la experiencia en campo"ai.

En el apartado anterior presentamos la metodología que utilizamos para la intervención a propósito del Plan Integral de Vida comunitario. Al tiempo que llevábamos a cabo el proceso de impulso al Plan Integral de Vida definiendo problemáticas, responsables, alternativas de solución y reconstruyendo parte de la historia reciente de la comunidad, surgieron inquietudes sobre nuestro lugar como profesionales que abanderaban la idea de los Planes Integrales Vida.

En el desarrollo del proyecto propusimos una serie de principios metodológicos como participación, flexibilidad, respeto y reconocimiento a la diversidad para la intervención en un espacio étnico particular, con lo que se buscábamos considerar la reflexividad ética antes de intervenir en una comunidad indígena. En relación con esos principios planteamos actividades con enfoque diferenciado pues considerábamos fundamental buscar la participación de "toda" la comunidad, como proponen algunas de las metodologíasaj para la elaboración de los Planes Integrales de Vida. Involucrarnos en la dinámica comunitaria implicó participar de las rutinas diarias de distintas personas y de la comunidad en general, con el objetivo de entender al Otro indígena que imaginábamos en el papel como un ser humano completamente diferente y extraño a nosotras.

No obstante, queremos enfatizar que nuestra intervención no se planteó como un mero conjunto de actividades orientadas a unas metas que se podían alcanzar y medir con determinados indicadores. Aunque inicialmente partíamos de supuestos idealizados sobre la noción de comunidad indígena (común y unidad) y la noción idealizada del Plan de Vida, en campo hubo una reflexión ética que se tradujo en una acción respetuosa enmarcada en un espacio (el resguardo indígena) como un tipo de organización política y administrativa, con unas rutinas culturales y un conjunto humano con una cosmovisión diferente y no por ello desigual o menos importante a la nuestra.

En el campo fue en donde pudimos acercarnos a ese Otro indígena que inicialmente nos desencantó al no coincidir con la figura del "buen salvaje", pero que después aprendimos a reconocer y valorar como un sujeto, un Otro cultural étnico, más próximo sin que por ello perdiéramos de vista su diferencia. En la convivencia 
cotidiana constante, que implicó el acompañamiento en la acción y la vinculación emotiva con su realidad, fuimos redescubriendo el ser indígena, y en lugar de juzgar su situación como "aculturación", empezamos a comprender la existencia de una identidad indígena contemporánea.

El Plan Integral de Vida fue la otra noción que desmitificamos. Inicialmente para nosotras era la panacea de la participación, el mecanismo para poner en diálogo a comunidades y Estado, que además debía surgir de toda la comunidad, pues representaba el proyecto de la vida de una etnia. Lo primero que encontramos fue que el tema no era de interés de todas las comunidades y que quienes abanderaban el proceso eran las organizaciones, ya que son quienes están más relacionadas con el lenguaje abstracto del reconocimiento, de la diversidad y el respeto a la diferencia, así como con las obligaciones y compromisos del Estado al respecto. Fue entonces cuando cuestionamos nuestro papel como promotoras de un discurso de la democracia participativa, dirigida desde afuera no por indígenas, basada en una idea de comunidad indígena como grupo compacto, altamente organizado y jerarquizado, en torno a la causa indígena reivindicativa, sin tener en cuenta que en la realidad es imposible encontrar un colectivo humano que funcione como un todo orgánico.

La cuestión era que estábamos en una comunidad que en su interior albergaba otra diversidad (tikunas, cocamas, yaguas, huitotos y mestizos), con aproximadamente 430 habitantes; en donde sólo se hablaba en lengua materna (indígena) en el espacio familiar, ya que el español había sido el idioma para poner a dialogar a sus habitantes; una comunidad que a pesar de su diversidad interna tenía una conciencia étnica clara hacia el exterior con la que negociaba e interactuaba, partiendo de su condición de indígenas, y desde la cual postulaban sus demandas de derechos frente a otros actores estatales y de la sociedad civil.

Para la mayoría de personas con quienes propusimos actividades el Plan Integral de Vida no era una cuestión relevante, la cual nos hicieron entender al momento de pensar en un grupo que estuviera al frente del proceso de sistematización del trabajo que conjuntamente habíamos adelantado. Es en ese momento que reconocimos e incorporamos en la práctica el otro componente de la ética en la intervención, es decir, el componente político.

La reflexión sobre nuestra noción ideologizada de los Planes Integrales de Vida como máxima de la participación, empoderamiento y reconocimiento étnico, y de las comunidades indígenas como un todo estático y homogéneo e intensamente 
cohesionado, nos llevó a reconocer y examinar los intereses políticos que implicaba esta experiencia en el campo ${ }^{\mathrm{ak}}$. Y decidimos entonces que con nuestra intervención no podíamos otorgarnos ad hoc la labor de impulsar el Plan Integral de Vida de la comunidad, ya que esta es una labor que incumbe únicamente a las comunidades y a sus organizaciones. Así, en medio de la práctica, redireccionamos nuestro rol profesional y decidimos aportar en el proceso de construcción del Plan Integral de Vida en el nivel de la recopilación de información referida a unas temáticas particulares: la construcción de censos, la identificación de problemas y la escritura de proyectos para la gestión de recursos.

Queremos subrayar que la intervención no puede desligarse de un ejercicio reflexivo constante sobre el carácter ético-político -además del conceptual y metodológico- que la sustenta, ya que al optar por la reflexividad valorativa profesional podemos asumir responsabilidades sobre la intervención en contextos étnicamente diversos. Este conocimiento sobre la implicación ético-política

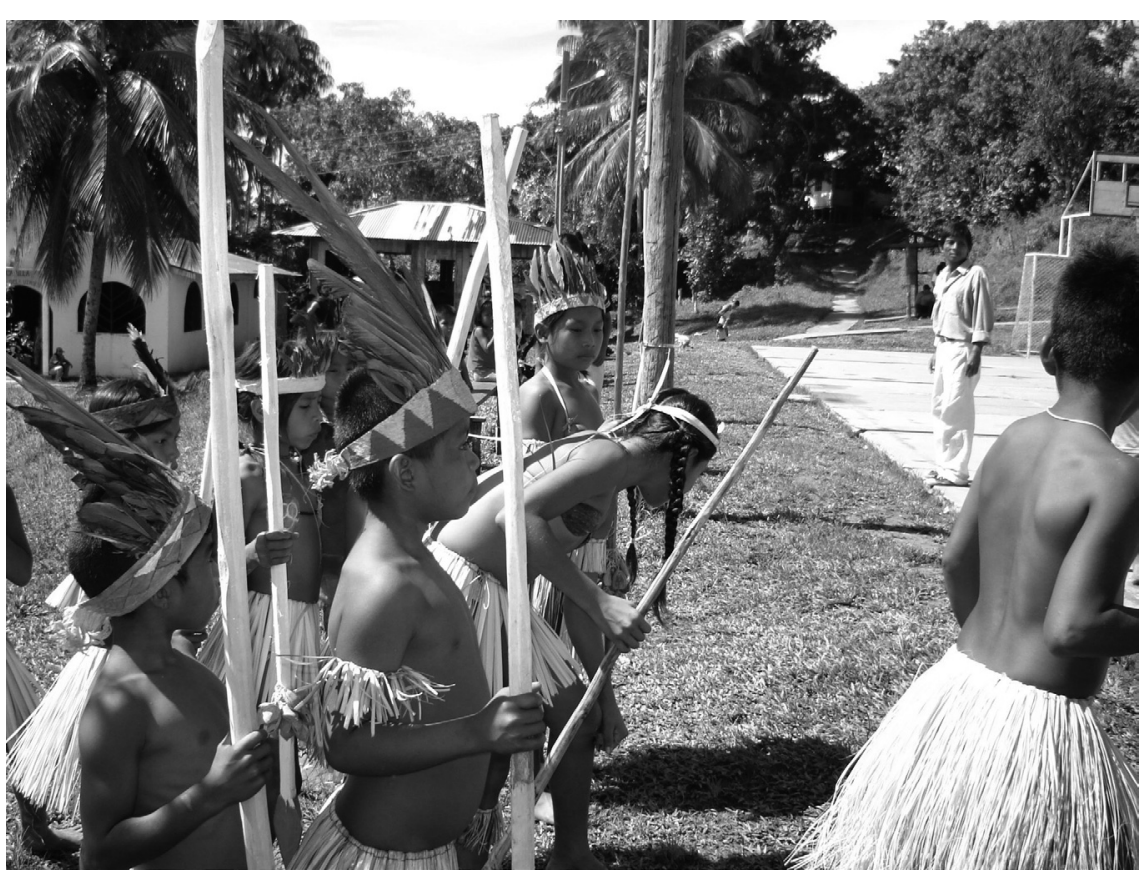
nos permite/obliga evitar caer en propuestas homogenizantes que promueven una imagen cristalizada del Otro indígena, en la cual se enmascaran prejuicios como pérdida cultural y el blanqueamiento, o la idea de que democracia es igual a participación en dinámicas locales de gestión de recursos.

La consideración de lo ético y lo político en la intervención también nos permite tener la posibilidad de tomar distancia de la cultura del Otro (por ejemplo,. en relación con la poca valoración que existe respecto de la posición de las mujeres, niños y niñas frente a las problemáticas internas), ya que como proponíamos en el apartado sobre la dimensión conceptual, entender no significa compartir y aceptar situaciones en las que existe un desentendimiento de la opinión de un conjunto importante de la comunidad.

En última instancia, hacemos un llamado de atención para que en la intervención se tenga en cuenta que el sujeto étnico al cual nos acercamos como profesionales nunca va a ser un sujeto prístino que algunos quieren retratar y conservar, sino que se trata de un sujeto étnico e histórico particular cuya identidad es 
dinámica, que por lo general es habitante de geografías consideradas de frontera, territorios salvajes y tierras de nadie ${ }^{a l}$, y que ha establecido relaciones con una sociedad mayoritaria -no nos atrevemos a llamarla occidental- que lo excluye de la justicia social y de los derechos humanos, muchas veces amparada en el respeto a la diversidad y la preservación cultural; que además le obliga a ser diferente y demostrarlo, a la vez que apuntala en la imposibilidad de llevar esa diversidad al límite de una autonomía fuerte ${ }^{a m}$. Consideramos entonces que, aunque es en este contexto (sociedad mayoritaria) donde se legitima nuestro quehacer profesional, es posible tomar distancia a partir de la reflexividad, en favor de la coherencia entre los componentes: conceptual, metodológico y éticopolítico de la intervención profesional intercultural.

\section{Conclusión}

El análisis anterior se centró en tres aspectos de la intervención. En primer lugar se propuso la dimensión conceptual, que se refiere a la importancia de tener nociones conceptuales dinámicas o en constante evaluación y relectura respecto a la intervención social, el Trabajo Social y los Planes de Vida con el fin de dar acento a la cuestión de la reflexividad conceptual sobre la acción intercultural, la cual incluye la reflexividad sobre un contexto cultural particular en relación con los elementos que propone Esteban Ruíz: elemento cultural, elemento discursivo y al elemento de las relaciones de poder. En segundo lugar quisimos llamar la atención sobre la dimensión metodológica de la intervención, en la cual existe un importante componente práctico. Por último, la dimensión ético-política, que guía desde niveles abstractos y concretos la intervención social, además de sugerir una reflexividad personal y profesional de las/os intervinientes que deciden actuar en un espacio étnico-cultural particular.

En relación con la cuestión de la intercultural de la intervención desde Trabajo Social, proponemos que es una especificidad sobre la que es pertinente reflexionar, en la medida que "dota nuestra praxis de un sentido crítico, de reflexionar sobre el papel de mediación que ejercemos entre sistemas culturales diferenciados"an, para movilizarnos en torno a la transformación de la noción de diversidad como justificación de la desigualdad social con el argumento del respeto a la particularidad cultural. Del mismo modo permite reconocer las transformaciones racionales y emocionales que operan en nosotras mismas como sujetos de la mediación en relación con el Otro, sujeto de la intervención, pero también en relación con el sí mismo profesional y personal. 
Finalmente, invitamos a colegas, estudiantes y profesionales de Trabajo Social a reflexionar juiciosamente sobre el tema del encuentro cultural con la diferencia -ya sea en el registro de lo étnico, el género, la generación o la opción sexual- y la intervención. Esto con el objetivo de construir lo que algunas/os autoras/es denominan "intervención sensible a la diferencia", no sólo para intentar entender cómo es la cuestión de lo cultural en "los Otros", sino también en el "sí mismo" involucrado en la intervención profesional.

\section{BIBLIOGRAFIA}

AROCHA RODRÍGUEZ, Jaime y MORENO TOVAR, Lina del Mar, (2007) "Andinocentrismo, salvajismo y afro-reparaciones", En: MOSQUERA ROSEROLABBÉ, Claudia y BARCELOS, Luiz Claudio (2007). Afro-reparaciones: Memorias de la Esclavitud y Justicia Reparativa para negros, afrocolombianos y raizales, Bogotá, Universidad Nacional de Colombia-FCH-CES-GEA. p. 587-614

BELLO, Martha y MILLÁN, Constanza (2006). "La intervención institucional en contextos culturalmente distintos: lógicas en tensión y contradicción.". En Revista Palimpsestus Bogotá, Universidad Nacional de Colombia p. 250-260.

GARCÍA CASTAÑO, Javier y BARRAGÁN RUIZ-MATAS, Cristina (2004). "Mediación intercultural en sociedades multiculturales: Hacia una nueva conceptualización". Revista Portularia No. 4. www.portularia.com. Fecha de consulta 21 de agosto 2006. p. 123-142

GARCÍA JORBA, Juan (2000). Diarios de campo. Madrid: Cuadernos Metodológicos 31-CIS

GROS, Cristian. (2004)” ¿Cuál autonomía para los pueblos indígenas de América Latina?" En: AROCHA, Jaime. Utopía para los excluidos. Bogotá, Universidad Nacional de Colombia. p. 205-230.

LORENTE MOLINA, Belén (1999). "La diversidad cultural y la práctica profesional del Trabajo Social: inmigración y relaciones interétnicas en espacios multiculturales". En: LORENTE, Belén y ZAMBRANO, Carlos. Estudios introductorios en relaciones interétnicas. Bogotá, Universidad Nacional de Colombia.

MALAGÓN BELLO, Edgar (2000). "Las relaciones de Bienestar social y los campos de intervención del Trabajo Social" En Revista del departamento de Trabajo Social, 
Bogotá, No. 2 p. 15-23

.(2003), "Trabajo Social: Ética y Ciencia." En Revista del departamento de Trabajo Social, Bogotá, No. 5 p. 12-22.

MOSQUERA, Claudia (2006). "Pluralismos epistemológicos: hacia la valorización teórica de los saberes de acción. Una reflexión desde la intervención social a la población afrocolombiana desplazada." En: Revista Palimpsestus Bogotá, Universidad Nacional de Colombia. p. 262-276

RICHMOND, Mary (2005). Diagnóstico Social. Madrid, Siglo XXI.

ROMERO BACHILLER, Carmen (2003) "Los desplazamientos de la "raza": de una invención política y la materialidad de sus efectos", En: Revista Política y Sociedad. Madrid, Vol. 40, No.1, 113.

ROZAS PAGAZA, Margarita (2001). La Intervención Profesional en relación con la cuestión social: El caso del Trabajo Social. Buenos aires, Espacio.

RUÍZ BALLESTEROS, Esteban (2005). Intervención social: cultura, discursos y poder. Madrid, Talasa.

SÁNCHEZ VIDAL, Alipio (1999). Ética de la intervención social. Barcelona, Paidos.

SERJE, Margarita (2005). El revés de la Nación: territorios salvajes, fronteras y tierras de nadie. Bogotá, Universidad de los Andes.

VÁZQUEZ AGUADO, Octavio (2002). "Trabajo Social y Competencia Intercultural". Revista Portularia No. 2 www.portularia.com. Fecha de consulta 21 de agosto 2006. p. 125-138

\section{BIOGRAFIA}

\section{RUBY ESTHER LEON DIAZ}

Trabajadora Social. Universidad Nacional de Colombia. Actualmente vinculada como coinvestigadora del proyecto "Acciones Afirmativas para afrocolombianos, negros y raizales: un paso hacia la Justicia Reparativa" adscrito al Grupo de Estudios Afrocolombianos de la Universidad Nacional de Colombia-sede Bogotá 


\section{NOTAS AL PIE}

a Sin intención de restar importancia política a los pueblos indígenas en el presente artículo me referiré a las comunidades indígenas para denominar a los resguardos que se asientan en esta zona del país. La denominación "comunidad" da cuenta de la manera en que los mismos miembros de los resguardos denominan a sus asentamientos (Resguardos).

b Comunidad indígena ubicada en el Trapecio Amazónico de Colombia, a 56 kilómetros de Leticia.

c MALAGÓN BELLO, Edgar (2003), "Trabajo Social: Ética y Ciencia." En Revista del departamento de Trabajo Social, Bogotá, No. 5 p.12-22

d El maestro Jaime Arocha y Lina del Mar Moreno denominan andinocentrismo al "modelo de nación que ideó la élite del siglo XIX a partir de la idea de que el ámbito de la civilización se daba en las zonas temperadas de los Andes, desde donde bajaba a las fronteras tórridas y salvajes". Ver AROCHA RODRÍGUEZ, Jaime y MORENO TOVAR, Lina del Mar, (2007) "Andinocentrismo, salvajismo y afro-reparaciones", "en" MOSQUERA ROSERO-LABBÉ, Claudia y BARCELOS, Luiz Claudio (2007). Afro-reparaciones: Memorias de la Esclavitud y Justicia Reparativa para negros, afrocolombianos y raizales, Bogotá: Universidad Nacional de Colombia-FCH-CES-GEA, p. 587

e RICHMOND, Mary (2005). Diagnóstico Social. Madrid: Siglo XXI, p. 449. El resaltado en cursiva es de la autora.

f VÁZQUEZ AGUADO, Octavio (2002). "Trabajo Social y Competencia Intercultural". Revista Portularia No. 2 www.portularia.com. Fecha de consulta 21 de agosto 2006, p. 125

g MALAGÓN BELLO, Edgar (2000). "Las relaciones de Bienestar social y los campos de intervención del Trabajo Social" En Revista del departamento de Trabajo Social, Bogotá, No. 2 (p.1523)

h VÁZQUEZ AGUADO, op.cit, p.130

i VÁZQUEZ AGUADO, ibid, p.130

j RUÍZ BALLESTEROS, 2006, 148 (La cita debe ser completa como se ha realizado antes)

k RUÍZ BALLESTEROS, ibid, p.160 y 168

1 VÁZQUEZ AGUADO, op.cit, p.135)

m GARCÍA CASTAÑO, Javier y BARRAGÁN RUIZ-MATAS, Cristina (2004). "Mediación intercultural en sociedades multiculturales: Hacia una nueva conceptualización". Revista Portularia No. 4. Www.portularia.com. Fecha de consulta 21 de agosto 2006; LORENTE MOLINA, Belén (1999). "La diversidad cultural y la práctica profesional del Trabajo Social: inmigración y relaciones interétnicas en espacios multiculturales.” En LORENTE, Belén y ZAMBRANO, Carlos. Bogotá (2002). Estudios introductorios en relaciones interétnicas. Universidad Nacional de Colombia; y VÁZQUEZ AGUADO, op.cit, p. 125-138.

n CASTILlEJO CUELLAR, Alejandro. Poética del Otro: para una antropología de la guerra, la soledad y el exilio interno en Colombia. Bogotá: ICANH-COLCIENCIAS, 2000.

o Lo étnico-racial se considera como una condición cultural que marca diferencias entre los sujetos que interactúan en un entorno social. Aunque se considera que científicamente están superados los discursos sobre razas, hoy más que nunca la "raza" se constituye "en una poderosa ficción reguladora mediante la que determinadas diferencias pasan a ser significadas en determinados contextos, estabilizando jerarquías y confiriendo ciertas características encarnadas como legitimadoras de opresiones y exclusiones, privilegios y dominaciones". ROMERO BACHILLER, Carmen (2003) "Los desplazamientos de la "raza": de una invención política y la materialidad 
de sus efectos", "en" Revista Política y Sociedad. Madrid, Vol. 40, No.1, 113. Evidencia de este proceso es la "racialización", a partir de la cual tales diferencias se vuelven significativas marcando determinados cuerpos, espacios y representaciones.

p ANGÉL, Rocío, PEREZ, Gilma, RICO, Martha, SANDOVAL, Rosa y VARGAS, Cecilia (1974). Acción de Trabajo Social en el campo indígena. Guapi-Cauca: Universidad Social Católica de La Salle, Prefectura Apostólica de Guapi,p.15

q BELLO, Martha y MILLÁN, Constanza (2006). "La intervención institucional en contextos culturalmente distintos: lógicas en tensión y contradicción.". En: Revista Palimpsestus Bogotá, Universidad Nacional de Colombia p. 250-260.

r MOSQUERA, Claudia (2006). "Pluralismos epistemológicos: hacia la valorización teórica de los saberes de acción. Una reflexión desde la intervención social a la población afrocolombiana desplazada." En: Revista Palimpsestus Bogotá, Universidad Nacional de Colombia p. 262-276

S MOSQUERA, ibíd., p. 268

t Entre julio de 2005 y abril de 2006 gracias al apoyo financiero de la ONG Tropenbos y el apoyo académico en campo de la profesora Luz Marina Donato, junto con mi colega Nini J. Cortez Perdomo se desarrolló el proyecto "Impulso a la formulación del Plan Integral de Vida de la comunidad indígena de Mocagua en el Trapecio Amazónico Colombiano". Posteriormente en el trabajo de análisis y reflexión sobre el Trabajo Social Intercultural a partir de la experiencia práctica de trabajo con una comunidad indígena la profesora Claudia Mosquera Rosero-Labbé guió el proceso de reflexión y escritura de mi trabajo de monografía "Propuesta para la compresión del Trabajo Social Intercultural: Reflexiones sobre el Traba Social en una comunidad indígena del Trapecio Amazónico Colombiano. Trabajo de monografía para optar al título de trabajadora social. Universidad Nacional de Colombia, Bogotá, 2006

u Acuerdo 169 de 1989 de la OIT

V El acento sobre el carácter colectivo de esta experiencia parte del supuesto de que toda aprehensión de la realidad es un producto social aunque pase por una interpretación subjetiva individual. Es en el espacio de la intervención en donde se ponen en juego la razón y la emoción; y en donde surgen -a diferencia del conocimiento científico universal e irrefutable-, los saberes como "epistemes locales (...) altamente variables y no universales; entretejidos con prácticas dinámicas; que involucran una negociación o conversación continua con una situación que se desea modificar". Wynne en MOSQUERA, op.cit, p.266.

Desde la noción de saberes el presente trabajo se inscribe como una co-producción (Mosquera 2006), más que como una creación netamente individual, por tanto, las reflexiones expresadas en este documento se plantean en co-autoría: con algunos miembros de las comunidades que nos acogieron y compartieron con nosotras sus reflexiones; con las ONGs que como organizaciones locales e internacionales brindaron orientación y apoyo; con los conocimientos académicos y profesionales adquiridos en la Universidad; por su puesto con mi colega y amiga Nini Cortez Perdomo en los meses de estadía en este lugar geográfico y también de vuelta en la ciudad de Bogotá. Y con la profesora Claudia Mosquera Rosero-Labbé quien acompañó y guió una parte importante de mi reflexión sobre el Trabajo Social Intercultural a partir de la experiencia que viví en el Resguardo Indígena de Mocagua. Por eso se busca no hacer uso del impersonal como estilo de escritura, sino la primera persona del plural.

w LORENTE MOLINA, 1999; VÁSQUEZ AGUADO, 2002; GARCÍA Y BARRAGÁN, 2004

x RUÍZ BALLESTEROS, op.cit, p.114-139

y SERJE, Margarita (2005). El revés de la Nación: territorios salvajes, fronteras y tierras de nadie. Bogotá: Universidad de los Andes, p. 114

z RUÍZ BALLESTEROS, op.cit, p. 160, el relatado en cursiva es nuestro

aa Cristian Gros critica esta noción, a la que denomina de carácter "ligero", considerando que así se reduce la autonomía a "un reconocimiento y una institucionalización de formas de organización social, económica y

Palobrano. 8 Agosto/2007 
política, que las comunidades indígenas desarrollan hoy por hoy (...) en el ámbito de las comunidades locales o de los municipios". El planteamiento de Cristian Gros nos permite afirmar que en los PLAN INTEGRAL DE VIDA existe una noción de autonomía ligera, ya que el Estado entra a establecer lineamientos y a institucionalizar prácticas "autónomas" de las comunidades indígenas en cuanto al manejo y distribución de sus recursos, lo que se evidencia por ejemplo en la determinación de los sectores de inversión de recursos propios de las comunidades, para conferirles un carácter formalizado por la legislación nacional, en armonía con los planes de desarrollo que se renuevan con cada gobierno. GROS, Cristian. "¿Cuál autonomía para los pueblos indígenas de América Latina?"(2004) "En: AROCHA, Jaime (2004). Utopía para los excluidos... Bogotá: Universidad Nacional de Colombia, p.216-217

La reflexión sobre el derecho a la autonomía nos permite afirmar también que los PLAN INTEGRAL DE VIDA desde el Estado, se convierten en un mecanismo que permite a las comunidades indígenas de manera "ligeramente autónoma", traducir sus demandas en proyectos para acceder a los recursos, en la retórica del "... mejoramiento de las condiciones de vida, de trabajo y del nivel de salud y educación de los pueblos interesados, con su participación y cooperación... OIT. Convenio 169, 1989: articulo 7.

ab RICHMOND, op.cit; AYLWIN, Nidia (1976). Un enfoque operativo de la metodología del Trabajo Social. Buenos Aires: Humanitas.

ac ROZAS PAGAZA, Margarita (2001). La Intervención Profesional en relación con la cuestión social: El caso del Trabajo Social. Buenos aires: Espacio.

ad SÁNCHEZ VIDAL Alipio (1999). Ética de la intervención social. Barcelona: Paidos.

ae SÁNCHEZ VIDAL, ibid, p. 58

af Anotamos que es posible relacionar las tareas prácticas que propone Belén Lorente con las competencias culturales sobre la intervención intercultural, propuestas por Octavio Vázquez (2002). Esto sugiere la existencia de una coherencia en la reflexión sobre la intervención en tanto objeto de conocimiento y como mecanismo puntual de la acción. LORENTE MOLINA op.cit, p. 245

ag Ibid,, p. 246

ah Ibid, p. 83

ai Ibid, p. 194

aj Se han producido una serie de documentos que pretenden dar luces a las comunidades indígenas respecto de la forma de formular y poner en práctica los PIV. Son tres las metodologías que más circulan propuestas por la Organización Nacional Indígena de Colombia-ONIC, la Dirección General de Asuntos Indígenas-DGAI y la Defensoría del Pueblo. Todas se plantean como una guía flexible, elaborada con participación de algunos líderes indígenas en unas de manera más amplia y constante que otras..

ak SÁNCHEZ VIDAL, Op.cit.

al SERJE,op.cit.

am GROS, op.cit.

an LORENTE MOLINA, op.cit, p. 249 